\title{
A Neural Network System for Productivity Forecasting
}

\author{
Dr A. H. Boussabaine, Lecturer
}

The liverpool School of Architecture and Building Engineering, The University of Liverpool, P.O. Box 147, Liverpool L69 3BX, U.K.

\section{Introduction}

Forecasting construction operation productivity is experience- based due to the complexity involved according to experience, an estimator can intuitively adjust the standard rates in productivity books to estimate for an operation in given project conditions. However, such practices do not guarantee a consistent estimate due to the lack of a binding mechanism that relates the present case to past patterns. Mathematical and statistical models have been applied to productivity forecasting. However, the application of such model's has to be based on arbitrary assumptions to simplify the mathematics, which limits their use in complex situations. Mathematical models only deal with operation efficiency and rely on input from the user of operation attributes such as the labour skills. They themselves do not address the impacts of environment factors and incorporate such effects on a productivity estimate. Neural network and expert systems of factors affecting production output, available to the planner through the medium of the mico-computer, could be valuable tools by which to improve planning and productivity, and hence minimize the costs of construction.

Neural networks have the ability to learn and generalize from examples, to product meaningful solutions to problems even when input data contains errors or is incomplete, to adopt solutions over time to compensate for changing circumstances, to process information rapidly, and to transfer readily between computing systems. This important property of neural networks might enable the performance of the complex multi-attribute, nonlinear mapping for estimating construction operation productivity. Therefore, this research uses neural networks and expert systems as an alternative solution to the stated problem. A system to achieve this objective is being developed at the University of Liverpool. This system consists of an expert system acting as, a store of facts and, rule of thumb about environmental factors and a neural network acting as a learning brain for the whole system. 


\section{Factors Which Affect Productivity}

Construction productivity has received much attention and discussion within the industry in the past two decades. However, the factors that actually affect productivity at the site level continue to be the subject of considerable research. The quantification of the effect of these factors upon operation durations is also a very challenging research topic.

A reliable determination of the project operations productivity is one of the major concerns of planning engineers. This reliability is dependent upon the accuracy of estimating the effect of various productivity factors in the project environment. The common practice is that the effect of these productivity factors is considered intuitively depending upon the skill of the planners (Adrian 1987). For a more reliable forecast of project operations productivity estimation, this study introduces a systematic model that can simulate the expected occurrence of the productivity factors, explicitly analyze and quantify their combined effect, and incorporate it in the operations duration estimation equation. Such a systematic model can be used to generate additional management information such as sensitivity of an operation to specific productivity factors (Boussabaine 1991).

The theory underlying the model hypotheses is that a project's productivity and hence duration is affected by a number of productivity factors, that may lead to random or systematic disturbance of performance and hence project duration uncertainty. The cumulative effect of these disturbances is a deviation of duration time that could be estimated and mitigated. However, if these disturbances or variances can be mathematically or statistically estimated and discounted from estimated durations, one is left with a less variable activity duration. Therefore, activity durations and hence project duration, are a function of a number of productivity factors that reflect the environmental conditions of a project. If cause-effect relationships of these productivity factors are known, then activity durations can be predicted to a higher degree of accuracy and/ or productivity maximised by avoiding the underlying causes. It is the purpose of this paper to propose a methodology which combines expert systems technology, simulation and neural networks to forecast construction productivity and refine estimates of activity durations.

The most frequently mentioned factors affecting labour productivity are labour factors, constructibility factors, site factors, management factors, plant factors and legal factors (Adrain 1987, Ahiya et al. 1984, Mechanical 1987, Thomas et al. 1994).

\section{Architecture of The System}

The architecture of the prototype knowledge base consists of a knowledge based shell that provides both a development and delivery environment, a neural network shell that provides the learning and refinement of productivity factors and a microcomputer that provides the environment for system prototyping and consultation The general architecture of the system is shown in fig. 1. The system consists of the knowledge base, the inference engine, the control strategy, the explanátion facility, the user interface and the neural network module. The knowledge base contains facts and rules about factors 
which affect productivity, the inference engine provides the medium process, the end-user interface provides the medium to consult the system, and the explanation facility provides the responses to use query during a consultation. The control strategy consists of rules or procedures that provide overall control of the system, conclusions, and the system's output. The neural network module is integrated with the expert system to generalise from the output samples the relationships between the dependent and independent productivity factors. Data generated form the expert system is used to train a neural network generalizing from the samples the relationships between the dependent and independent production attributes. A supervised learning technique (back- propagation) is used to train the system with example input- output patterns. The mapping ability acquired by a trained network is stored in its structure and distributed within the connection weights. After the network

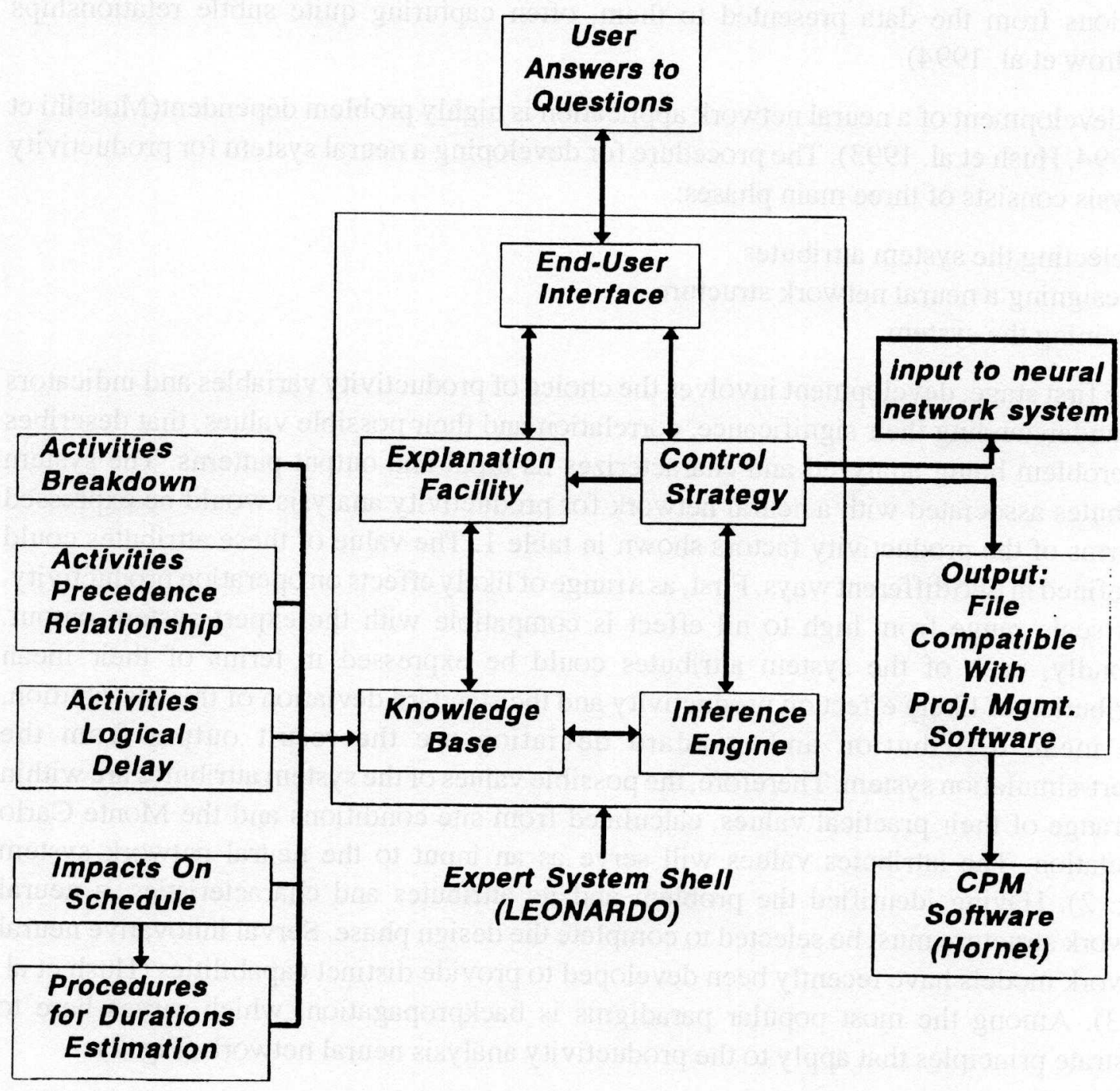

Figure 1. General Architecture of Prototype System 
system is fully trained the system would provide the required mapping function effectively in producing a productivity forecast for the construction operation in various site conditions.

\section{Conceptual Operation of the Neural Network System}

The output results from the expert system can be used in many ways. One of the possibilities is to use the output as an input for a neural network system. This section explores the background theory for developing an expert-neural system for productivity analysis.

A neural network is an implementation of an algorithm inspired by research into the brain (Hammerstrom 1994, Rumelhart et al. 1994). Neural networks are valuable on several counts. First, they are adaptive, they can take data and learn from it. Thus they infer solutions from the data presented to them, often capturing quite subtle relationships (Widrow et al. 1994).

The development of a neural network application is highly problem dependent(Moselhi et al. 1994, Hush et al. 1993). The procedure for developing a neural system for productivity analysis consists of three main phases:

a.) selecting the system attributes

b.) designing a neural network structure

c.) training the system.

In the first stage, development involves the choice of productivity variables and indicators and understanding their significance, correlation and their possible values, that describes the problem being analyzed and characterizes its input and output patterns. The system attributes associated with a neural network for productivity analysis would be expressed in terms of the productivity factors shown in table 1 . The value of these attributes could be defined in two different ways. First, as a range of likely effects on operation productivity. This scale range from high to nil effect is compatible with the expert system output. Secondly, each of the system attributes could be expressed in terms of their mean distribution of likely effect on productivity and the standard deviation of this distribution. The mean distribution and standard deviation are the result output from the expert-simulation system. Therefore, the possible values of the system attributes are within the range of their practical values, calculated from site conditions and the Monte Carlo simulation. The attributes values will serve as an input to the neural network system (Fig. 2). Having identified the problem and its attributes and characteristics, a neural network structure must be selected to complete the design phase. Serval innovative neural network models have recently been developed to provide distinct capabilities (Hush et al. 1993). Among the most popular paradigms is backpropagation, which serves here to illustrate principles that apply to the productivity analysis neural network (Fig. 2).

Conceptually, a backpropagation network is made from inter- connected nodes arranged in layers (Fig. 2). The input layer (productivity attributes) is passive, it merely receives the data patterns, that is the range of the attributes effect or the mean and standard deviation 
of the attributes, passing into the network. The number of input nodes consequently equals the number of measured data values (seven attributes). In a fully integrated system the inputs would be readings from the expert system output. The input layer broadcasts a pattern to all hidden nodes. These calculate a weighted sum of inputs as passed through a transfer function to generate an actual productivity forecast using productivity models (Fig. 2).

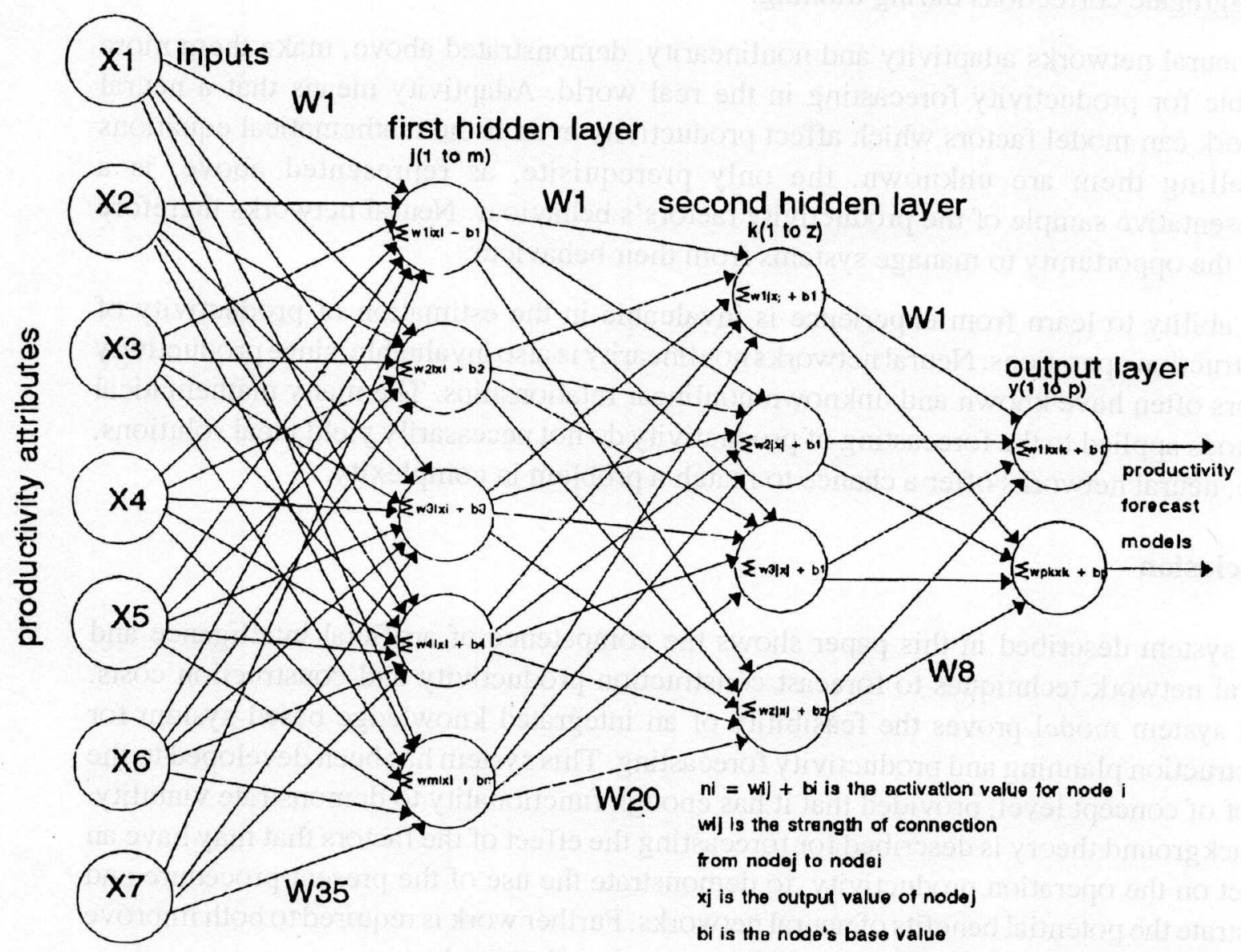

Figure 2. Productivity Forecasting Network Architecture

Each output node subsequently subtracts its productivity forecast from its desired result, which would be established as the upper and lower limits on productivity loss at the onset of a training session. This subtraction yields the output error.

The output nodes calculates the derivatives of the error with respect to the weights, subsequently passing these derivatives back to the hidden layer. In turn, each hidden node calculates the weighted sum of the error derivatives to find its contribution to the output error. Finally each hidden layer node and output layer node changes its weights according to predetermined mathematical methods, for example least squares, to reduce its error. 
The final forecast would be dictated by the relative levels of activation of the output nodes, so that the productivity calculation models represented by the node with the highest level of activation (effect) is used to forecast productivity loss.

In the learning stage, the designed network would be fed with a training set of data of attributes from the expert system output, and the desired output of the productivity forecast is estimated. This process is repeated until the network's overall accuracy is improved by the aggregate corrections during training.

The neural networks adaptivity and nonlinearity, demonstrated above, make them more suitable for productivity forecasting in the real world. Adaptivity means that a neural network can model factors which affect productivity even if the mathematical equations modelling them are unknown, the only prerequisite, as represented above, is a representative sample of the productivity factors's behaviour. Neural networks therefore offer the opportunity to manage systems from their behaviour.

This ability to learn from experience is invaluable in the estimation of productivity of construction operations. Neural networks nonlinearity is also invaluable, since productivity factors often have known and unknown nonlinear relationships. The many mathematical methods applied to the forecasting of productivity do not necessarily yield ideal solutions. Here, neural networks offer a chance to match a problem in complexity.

\section{Conclusion}

The system described in this paper shows the competence of artificial intelligence and neural network techniques to forecast construction productivity and construction costs. This system model proves the feasibility of an integrated knowledge based system for construction planning and productivity forecasting. This system has been developed to the proof of concept level, provided that it has enough functionality to demonstrate viability. A background theory is described for forecasting the effect of the factors that may have an effect on the operation productivity to demonstrate the use of the present procedure and illustrate the potential benefits of neural networks. Further work is required to both improve system performance and establish a wide range of production factors.

Consideration is also being given to the possibility of applying neural networks to other types of construction problems, such as resource optimization, site layout and risk analysis. 


\section{Appendix. References}

Adrian, J. J (1987). Construction Productivity Improvement, Elsevier Science Publishing Co., New York.

Ahuja, H. N., and Nandakumar, V. (1984). Enhancing reliability of project duration forecasts, Trans. Amer. Assn. Cost Engrs., Montreal, Canada, PE6-1,E6-12.

Bennett, J. and Ormorod, R. N. (1984). Simulation applied to construction projects, Construction Management and Economics, Vol. 2, 225.

Boussabaine, A. H.(1991). An expert system for construction planning and productivity analysis, thesis presented to the University of manchester, at U.K., in partial fulfilment of the requirements for the degree of Doctor of Philosophy.

Hammerstrom, D. (1993). Neural Networks at Work, IEEE Spectrum, June, 26-32.

Hush, D. R., et al. (1993). Progress in Supervised Neural Networks: What's New Since Lippmann. IEEE Signal Processing Magazine, January, 8-38.

Mechanical Contractors Association of America, (1987). Factors affecting productivity, Management Methods Bulletin, No. Co1.

Moselhi, O., et al. (1991). Neural Networks as Tools in Construction, J. Mgmt. Engrg., ASCE, 117(4), 606-625.

Rumelhart, D., et al. (1994). The basic Ideas in Neural Networks, Communication of the ACM, March, Vol.37, No.3, 87-91.

Thomas, H. R., et al, (1994). Forecasting Labour productivity Using Factor Model, J. Mgmt. Engrg., ASCE, 120(1), 228-239.

Widrow, d., et al. (1994).NeuralNetworks: Applications in Industry, Business and Science, Communication of the ACM, March, Vol.,37, No.3, 93-105. 